\title{
Cloning, Characterization and Properties of Plasmids Containing CGG Triplet Repeats from the FMR-1 Gene
}

\author{
Miho Shimizu', Robert Gellibolian', Ben A. Oostra ${ }^{2}$ and \\ Robert D. Wells ${ }^{1 *}$
}

\author{
${ }^{1}$ Institute of Biosciences and \\ Technology, Texas A \& $M$ \\ University, Department of \\ Biochemistry and Biophysics \\ Texas Medical Center, 2121 \\ W. Holcombe Houston \\ TX 77030-3303 USA \\ ${ }^{2}$ Department of Clinical \\ Genetics, Erasmus University \\ Rotterdam, P.O. Box 1738 \\ 3000 DR Rotterdam \\ The Netherlands
}

\begin{abstract}
The FMR-1 gene for the human fragile- $X$ syndrome, a mental retardation disease inherited by non-Mendelian transmission, contains a genetically unstable CGG region in the 5' non-translated region. The severity of the disease is correlated with the length of the CGG tract. The cloning of 28 stable plasmids containing (CGG) ${ }_{n}$ inserts (where $n=6$ to 240 ) with different extents and types of sequence interruptions (polymorphisms), and in different orientations was accomplished by three strategies in Escherichia coli. Some shorter tracts were prepared by the direct cloning of synthetic oligonucleotides, and longer runs were clones of multimers of $(\mathrm{CGG})_{81},(\mathrm{CGG})_{11} \mathrm{AGG}(\mathrm{CGG})_{60} \mathrm{CAG}(\mathrm{CGG})_{8}$, from a cDNA from a fragile-X patient or from expansions or deletions of these sequences in E. coli. The genetic stability of the inserts, especially for the longer tracts, was dependent on the sequence length, the presence of polymorphisms, the host cell genotypes, the orientation of the inserts in the vector and the position of cloning in a vector. Two-dimensional agarose gel electrophoresis studies on fully methylated and on non-methylated plasmids as well as chemical probe studies revealed the absence of underwound structures or accessible base-pairs. These DNAs enable a range of genetic and biochemical investigations into the molecular basis of the fragile- $X$ syndrome.
\end{abstract}

(C) 1996 Academic Press Limited structure

\section{Introduction}

Several human hereditary neuromuscular and neurodegenerative diseases have been linked to variations in the length of CTG and CGG triplet repeats (TR) within their genes (reviewed by articles in Davies \& Warren, 1993). The fragile-X syndrome, an inherited mental retardation disease with several physical abnormalities, affects approximately one in 2000 males and is X-linked and carried by normal carrier (or transmitting) males whose daughters can have affected offspring

Present address: M. Shimizu, Genome Science Laboratory, Institute of Physical and Chemical Research (RIKEN), Tsukuba Life Science Center, 3-1-1 Koyadai, Tsukuba, Ibaraki 305, Japan.

Abbreviations used: TR, triplet repeat; 2-D, two-dimensional. (reviewed by Nelson, 1993). The penetrance of the disease increases with each generation, suggesting a non-Mendelian mode of transmission (anticipation). The genetically unstable region contains multiple CGG repeats (Kremer et al., 1991) within the mRNA of a fragile-X-associated gene (FMR-1; Verkerk et al., 1991; Fu et al., 1991). Normal individuals show heterogeneity at this site containing between six and 60 copies of the CGG triplet with an average of 29 repeats. The normal repeat contains the sequence (CGG) ${ }_{10}$ AGG(CGG) ${ }_{9}$ AGG(CGG), (Verkerk et al., 1991). Carrier (asymptomatic) males have a premutation between 60 and 200 copies of the repeat. Individuals with a full mutation, greater than 200 copies of the repeat, typically show symptoms of the fragile- $X$ syndrome. Expansion may occur during embryonic development, between five and 20 days post conception (Reyniers et al., 1993; Wohrle et al., 1993). 
The TR is located within the non-translated region of the FMR-1 gene. In affected males with greater than 200 copies of the repeat, there is no expression of the FMR-1 gene, which may be the result of methylation of a CpG island about $200 \mathrm{bp}$ $5^{\prime}$ to the CGG repeat (Yu et al., 1992; Sutcliffe et al., 1992; Hornstra et al., 1993). Methylation of this FMR-1 CpG island has been observed in fragile-X patients (Bell et al., 1991). The CGG repeat is completely methylated when expanded and the TR loses at least one AGG triplet, forming a longer perfect CGG repeat region. The FMR-1 gene is an RNA binding protein (Ashley et al., 1993; Siomi et al., 1993), although its biological role remains to be established. Expansion of CGG TR has been associated also with the FRAXE and the FRA11B loci (Knight et al., 1993; Jones et al., 1995).

The right-handed $B$ conformation of DNA in the Watson-Crick double helix ( $\sim 10.4 \mathrm{bp} /$ right-handed turn) is believed to be the principle structure in living cells. However, under appropriate conditions (sequence, negative supercoil density, ionic conditions, etc.), several types of non-B conformations have been well characterized. These structures exist in short segments at defined loci. For example, cruciform structures form in negatively supercoiled DNA within regions of inverted repeat symmetry (Sinden, 1994; Sinden \& Wells, 1992). $\mathrm{Z}$-DNA is formed at regions of alternating purine and pyrimidine sequences, such as (GT) $n_{n}$ or $(\mathrm{GC})_{n}$, under physiological conditions in negatively supercoiled DNA. Also, alternating AG sequences and other polypurine-polypyrimidine regions that contain mirror repeat symmetry form various intramolecular triple-helical isomers in which one half of the tract unpairs and one strand hydrogen bonds utilizing Hoogsteen base-pairing in the major groove of the DNA duplex. Other alternative conformations are known to exist with simple repeating sequences including nodule DNA, parallel DNA, tetrastranded telomere DNA structures, bent DNA, and slipped structures (Sinden, 1994; Sinden \& Wells, 1992; Wells, 1996). All of these alternative conformations were well characterized in vitro and several were shown to exist readily in living bacterial cells and in eucaryotes (Sinden \& Wells, 1992; Lukomski \& Wells, 1995).

Whereas these structural studies were conducted on a variety of simple sequences, few investigations have been conducted on TR sequences cloned into plasmids. Inserts containing long CGG repeats have been especially difficult to clone since they are prone to form deletion products (Gastier et al., 1995). Here we describe the cloning and characterization of a variety of lengths of CGG repeats containing different types of polymorphisms which may be used for future investigations including DNA structure, mismatch repair, mechanisms of replication and recombination related to expansion and deletion, and gene expression in prokaryotic and transgenic mouse systems.

\section{Results}

\section{Cloning and characterization of plasmids containing pure CGG inserts}

A family of plasmids containing pure (CGG) repeats, where $n$ ranged from 6 to 32, was prepared by a combination of standard cloning methodologies with synthetic oligonucleotides along with the procedures (Kang et al., 1995a) for generating expansions and deletions in vivo. Figure 1(a) outlines the method used for generating pRW3024 which contains $(\mathrm{CGG})_{24}$. The oligonucleotides were designed to produce a recombinant plasmid with CCG being on the leading strand with respect to the origin of replication of the pUC19-NotI.

pRW3024 was isolated and characterized by double digestion with SacI and HindIII by PAGE (see Materials and Methods). The analysis (Figure 1(b)) reveals intense bands (arrowheads) in lanes 2 and 3 corresponding to the expected full-length restriction fragments containing ten and 24 CGG repeats, respectively. Two interesting features are apparent. Firstly, the longer sequence, pRW3024, is more unstable (more deleted) in E. coli DH5 $\alpha$ compared to the shorter pRW3010; furthermore, the deletion products observed for pRW3024 are not a smear but rather resolve as distinct bands on the gel each differing from its neighbor by one CGG triplet. Secondly, in addition to deletions, expansion products are also observed which differ in length by one repeating unit. On the other hand, pRW3010 does not show the instability, i.e. expansion/deletion, observed for pRW3024, indicating that length is an important factor in the stability of the CGG TR.

To test whether the expanded/deleted bands are due to instability occurring within the TR, we eluted DNA from regions of the gel corresponding to the expansions and deletions and cloned the fragments into the SacI/HindIII region of the polylinker of pUC19-NotI. Shorter as well as longer clones containing six (pRW3006), eight (pRW3008), ten (pRW3010), 17 (pRW3017), 24 (pRW3024), 29 (pRW3029), 32 (pRW3032), 34 (pRW3675), 40 (pRW3681), 43 (pRW3679), 46 (pRW3677) and 49 (pRW3683) CGG repeats were characterized by sequencing. This is the first observation of expansion/deletion products occurring in integral multiples of the triplet repeats. Hence, the mechanism for this process must be complementary strand slippage as proposed previously (reviewed by Wells \& Sinden, 1993).

\section{Stability of (CGG) $)_{24}$ in different host cells}

The stability of a pure tract of (CGG) ${ }_{24}$ in different host cells posed a challenge for obtaining a homogeneous insert preparation. The propagation of CGG TR sequences in E. coli DH5 $\alpha$ provided an effective venue to create expansion as well as deletion products, but did not allow for the 
stabilization needed to generate a homogeneous length of repeat. However the best host cells for the stabilization of pure $(\mathrm{CGG})_{24}$ repeats is E. coli SURE (defining stability as the amount of full length insert as determined by PAGE). After 12 hours of growth ( 20 generations) in E. coli SURE the stability was $100 \%$, and in E. coli DH5 $\alpha, 65$ to $70 \%$. Hence, $\mathrm{DH} 5 \alpha$ is optimum for creating different lengths of derivative inserts whereas SURE is the preferred cell line for insert stabilization.

\section{Cloning strategy for long CGG repeats in plasmids}

The direct cloning of cDNA sequences from human patients has served as a source of investigative tools (Verkerk et al., 1991) but the majority of the triplet tracts are $<30$ repeats. Two other strategies may be exploited, the direct cloning of a synthetic DNA fragment (described above) and the in vivo expansion method in E. coli (Kang et al., 1995a). For structural studies, we wished to clone more than 200 CGG repeats. The synthesis and purification of such long tracts of CGG.CCG by chemical methods is intrinsically difficult. Application of the E. coli expansion methodology (Kang et al., 1995a,b) to CGG repeat sequences from the fragile- $X$ gene failed because of either less-frequent expansion of this particular TR in E. coli (Ohshima et al., 1996) or the difficulty in obviating deletions of the long tracts of non-interrupted CGG repeats, or both. In general, GC-rich sequences from microsatellites (Klysik et al., 1982), including CGG repeats (Gastier et al., 1995), are known to be difficult to clone. The instability of CGG repeat sequences in the human fragile- $X$ syndrome are caused by the loss of interruption (polymorphic) sequences (AGG; Eichler et al., 1994, 1995). We observed a similar instability of cloned non-interrupted CGG sequences in E. coli, especially for tracts longer than 30 triplets.

\section{Preparation of plasmids containing long repeats}

In fragile- $X$ patients, there is a correlation between the repeat length and the severity of the symptoms. For complete inactivation of the FMR-1 gene, it is known that the multiples of CGG should exceed more than 200. Thus, we wished to clone long CGG repeats $(>200)$ in a vector for structural studies which may lead to a better understanding of the molecular basis for the genetic instability of the repeats.

The DNA fragment containing a CGG repeat was isolated from RN2, a derivative of pT7Blue(R)

(a)

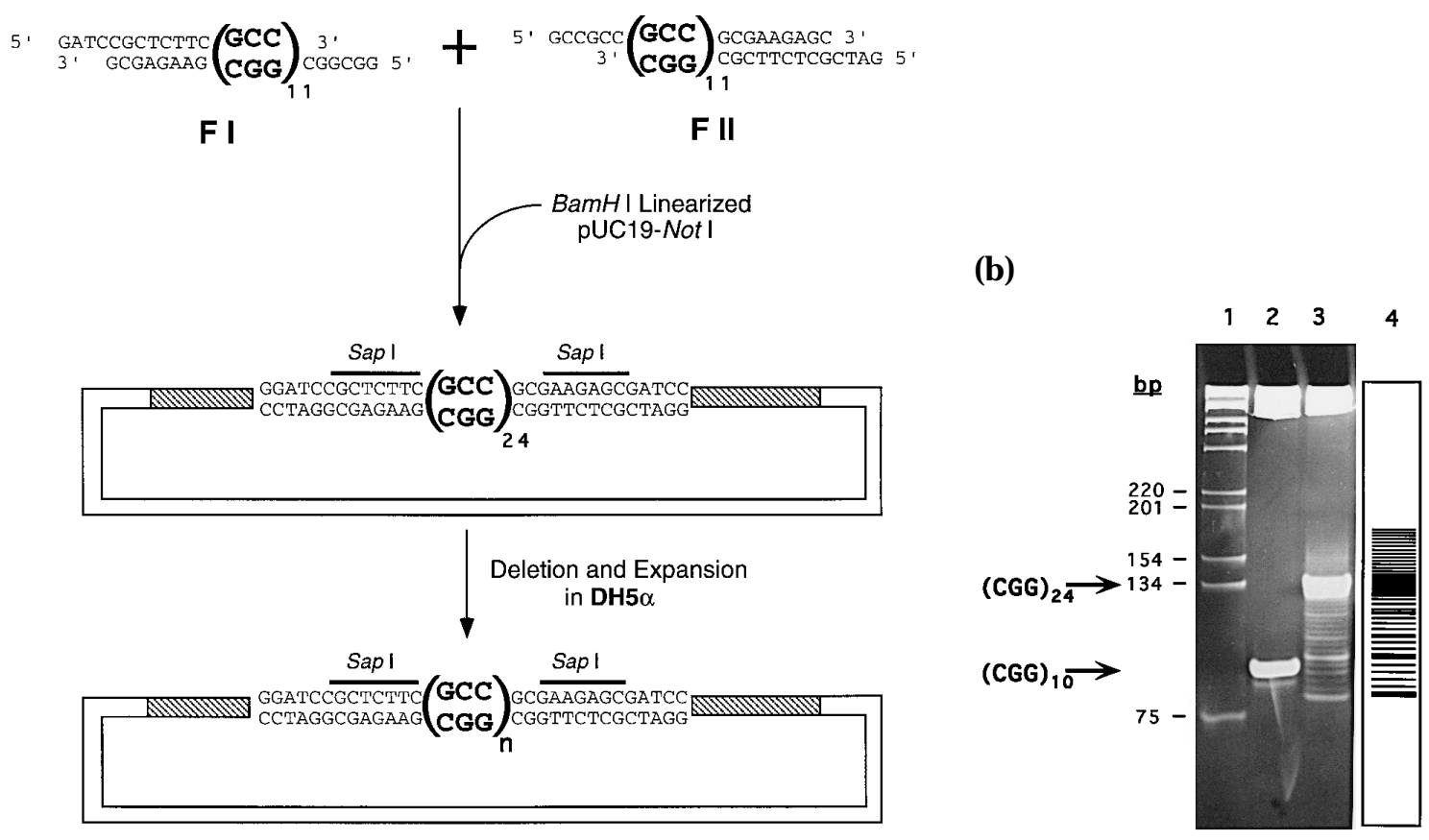

Figure 1. (a) Strategy for cloning stretches of pure CGG triplet repeats in pUC19NotI. Hatched vector regions designate the polycloning sites. (b) Polyacrylamide gel electrophoresis at $4^{\circ} \mathrm{C}$ of digests of plasmids containing short CGG tracts. Lane 1, 1 kb DNA ladder size markers; lanes 2 and 3, SacI/HindIII digests of pRW3010 and 3024, respectively, after propagation in E. coli DH5 $\alpha$ for 20 to 25 generations. The intense bands are the expected full-length fragments containing ten and 24 CGG repeats, respectively. The distinct bands below and above the full-length fragment with (CGG) 24 correspond to deletions and expansions within the triplet repeat. Lane 4, schematic reproduction of lane 3 (for clarity) showing the individual bands (in multiples of the triplet repeat) in the deleted and expanded areas. A 15 to $20 \mu \mathrm{g}$ sample of DNA was the optimal concentration which can be loaded onto the gel to visualize the individual bands corresponding to deletions and expansions without loss of resolution. 


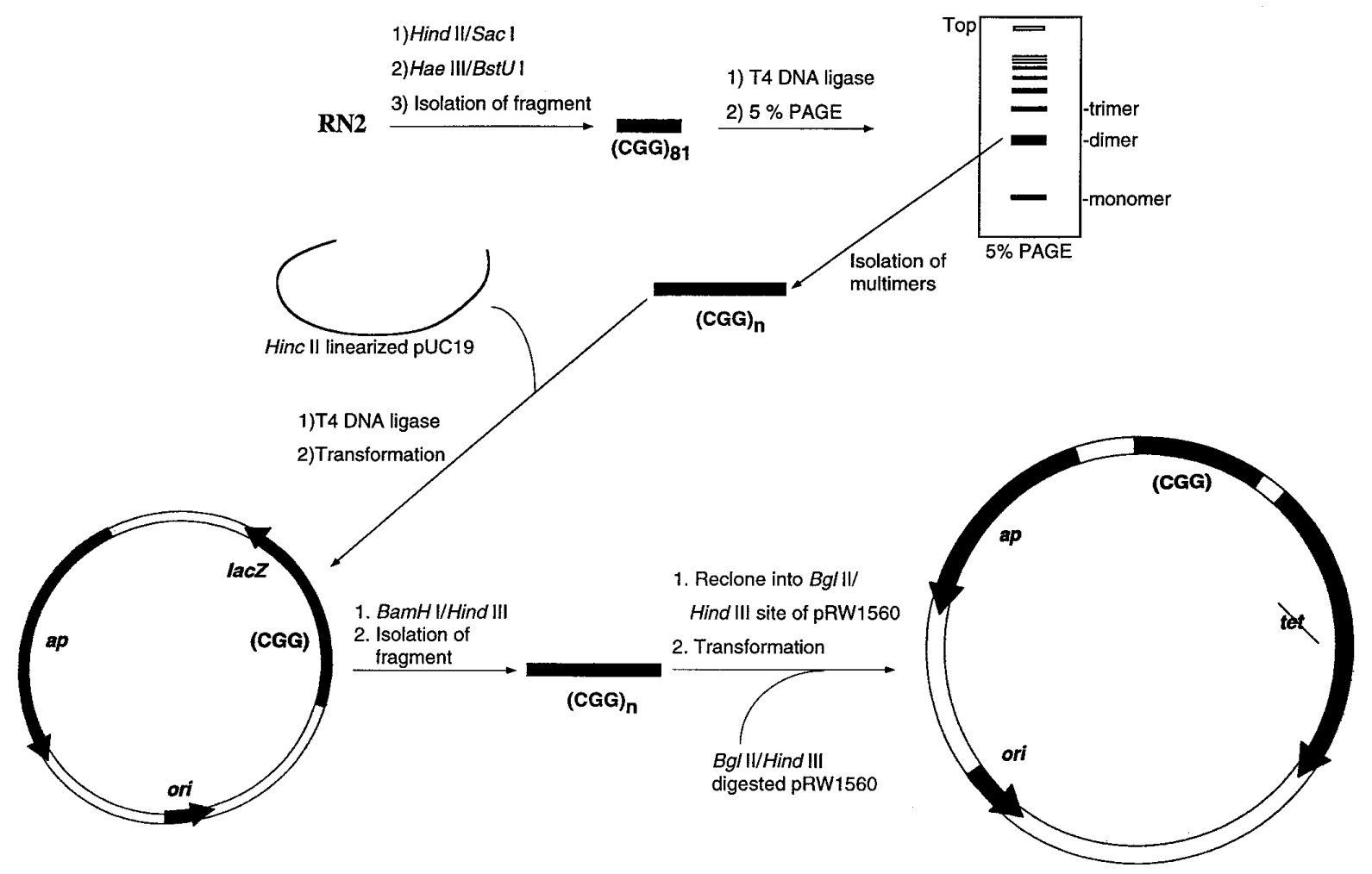

Figure 2. Cloning strategy for long CGG repeat sequences from the fragile-X gene. Ap, ampicillin; tet, tetracycline; ori, replication origin.

with a cDNA sequence from a fragile- $X$ patient containing (CGG) $)_{81}, \quad\left((\mathrm{CGG})_{11} \mathrm{AGG}(\mathrm{CGG})_{60} \mathrm{CAG}-\right.$ $\left.(\mathrm{CGG})_{8}\right)$, in the EcoRV site of the polylinker with only a small amount of flanking sequence $(5 \mathrm{bp}$ on the HaeIII side and no DNA on the other end; Figure 2). The resulting blunt-ended DNA fragment digested by Hae III and Bst UI was multimerized by T4 DNA ligase. Successful cloning was achieved by isolating the dimer from a $5 \%(\mathrm{w} / \mathrm{v})$ polyacrylamide gel and ligating it into dephosphorylated HincII linearized pUC19 followed by transformation into E. coli HB101 or SURE by electrotransformation. Restriction analysis of DNA from a single clone out of the nine analyzed revealed the presence of the dimer molecule of (CGG) ${ }_{81}$ as the major component (pRW3306) along with several smaller sized (deleted) CGG repeat sequences, including pRW3338 and pRW3339. Although E. coli SURE was the optimum strain used for stabilizing pRW3306, a large number of cell divisions caused the eventual instability (deletion) of the plasmid. Hence, the CGG-containing insert from pRW3306 was transferred into pBR322 (described below).

\section{Recloning (CGG) ${ }_{160}$ into a pBR322 derivative}

Prior studies (Jaworski et al., 1989) showed that sequences that were inherently unstable genetically due to their simple repeating tracts that could form non- $B$ structures were more stable when cloned into the promoter region of the tet gene of pBR322.
Hence, we recloned the dimer insert from pRW3306 into pRW1560 to assess the stability of the insert in this vector. pRW1560 is a pBR322 derivative with a $B g l \mathrm{II}$ site, which is compatible with BamHI ends, at the filled-in unique EcoRI site. pRW3306 was digested with Bam HI and HindIII at the polylinker site of the vector. The isolated fragment then was cloned into BglII/HindIII digested pRW1560 (dephosphorylated) in HB101. Agarose gel analyses of isolated plasmids showed that ten out of 16 clones contained a homogeneous (CGG) ${ }_{160}$ sequence. Thus, these data show that another unstable DNA sequence, a long CGG repeat, was stably cloned at an insertion-mutated tetracycline gene promoter of pBR322 as in the previous cases for Z-DNA and intramolecular triplexes (Jaworski et al., 1989).

\section{Cloning of a (CGG) ${ }_{240}$ insert}

Since we found that a long CGG repeat can be stably cloned into pBR322 as described above, we attempted to clone the (CGG) ${ }_{240}$ insert. A DNA fragment containing a trimer of $(C G G)_{81}$ was isolated and ligated into HincII-linearized (dephosphorylated) pUC19. Transformation was conducted using SURE cells. Three clones showed the presence of plasmids with inserts larger than that in pRW3306 but most isolates contained plasmids with smaller inserts.

Substantial instability of the inserts in E. coli was observed, especially for (CGG) ${ }_{240}$, in pUC19 


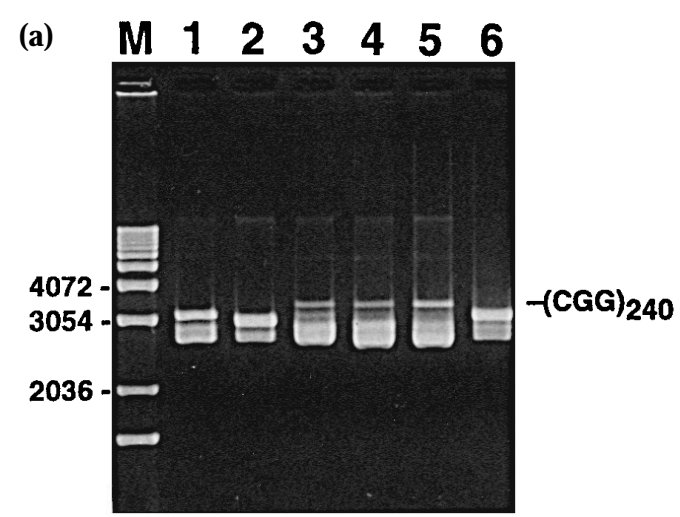

(b)

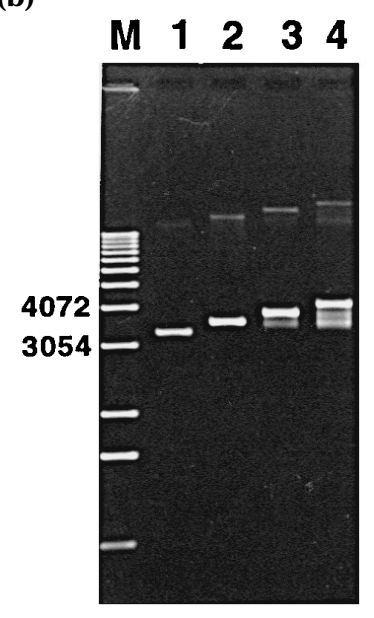

Figure 3. (a) Analysis of pRW3309 clones in E. coli SURE cells. Five transformants were grown in LB broth containing $70 \mu \mathrm{g} / \mathrm{ml}$ ampicillin at $37^{\circ} \mathrm{C}$ for ten hours. Lanes 1 to 5, DNA was extracted, linearized with SacI, and analyzed on a 1.5\% agarose gel; lane 6, SacI-digested pRW3306; lane M, size marker DNA. (b) Stability of CGG-containing pBR322 derivatives in E. coli HB101. Plasmids containing $(\mathrm{CGG})_{81},(\mathrm{CGG})_{160}$ and $(\mathrm{CGG})_{240}$, respectively, were prepared and run on a $1 \%$ agarose gel. Lane $M$, size marker DNA; lane 1, pBR322; lane 2, pRW3316; lane 3, pRW3308; and lane 4, pRW3310.

(Figure 3(a), lanes 1 to 5) compared to the dimer molecules (lane 6). Three out of five clones have a trace amount of the desired insert containing (CGG) 240 but most of the inserts were deleted into a distinct family of inserts with a smaller number of CGG repeats (lanes 3 to 5). In the case of two other clones (lanes 1 and 2), no full-sized plasmid DNA was observed. These plasmids were digested with Bam HI and HindIII to release the inserts which were fractionated on a $5 \%$ polyacrylamide gel. The uppermost bands, expected to be (CGG) $)_{240}$, were excised and the DNA fragments were purified (see Materials and Methods) and ligated into BglII/ HindIII-digested pRW1560. The ligation mixture was transformed into freshly prepared E. coli HB101 competent cells. Only one clone, pRW3310, contained a DNA fragment corresponding to (CGG) ${ }_{240}$ when analyzed on an agarose gel after digestion with HindIII and XbaI. pRW3310 was transformed again into HB101 to get a stable clone.

Since the inherent stabilization of larger CGG tracts in a pBR322 derivative (pRW1560) was established, we recloned the insert containing (CGG) ${ }_{81}$ into the same region of the vector as for pRW3310 and 3306. Figure 3(b) shows the stability of pRW3316, 3308 and 3310 which contain 81, 160 and 240 repeats of CGG, respectively, in pRW1560. At least twofold higher stability was obtained by recloning these CGG inserts into pBR322. Densitometric analysis of the gel showed that the expected-sized inserts were present in the following proportions; $100 \%$ for pRW3316 (lane 1), 77\% for pRW3308 (lane 2) and 53\% for pRW3310 (lane 3). On the other hand, these inserts are relatively unstable in pUC19; $100 \%$ for pRW3311, $46 \%$ for pRW3306 and 27\% for pRW3309.

In summary, the stability of the long (CGG) inserts was greatly enhanced by cloning into the promoter region of the tetracycline-resistance gene in pBR322 via subcloning through pUC19. Thus, repeats up to 240 units were stabilized in E. coli. Hence, it may be possible to clone even longer CGG repeat sequences (more than 240 repeats) into this region from a DNA fragment derived from human disease genes.

\section{Stability of inserts is determined by orientation and the presence of interruptions}

The frequency of expansion and deletion of CTG triplets is influenced by the direction of replication in E. coli (Kang et al., 1995a). The vectors PUC19 and pBR322, which were used for cloning in this study, have a unidirectional replication origin (ColE1). In the construction of pRW3311, pRW3306 and pRW3309, the ligation of CGG inserts into a HincII site of pUC19 could occur in either orientation because of the blunt ends. Interestingly, all inserts were found in only one orientation, namely, the CGG triplets were always in the leading strand for replication (orientation I) as revealed by dideoxy sequencing of the DNAs. For example, when pRW3311 was constructed, ten clones were analyzed for the orientation of the (CGG) $)_{81}$ insert in pUC19; all ten inserts were in orientation I (data not shown). Hence, the stability of the (CGG) $)_{n}$ repeats (where $n=81,160$ and 240) is orientation-dependent in E. coli as found for CTG (Kang et al., 1995a). Further studies described below confirm this conclusion.

To study the effect of cell division on the instability of CGG repeats, we constructed plasmids in which (CGG) ${ }_{160}$ was inserted in both orientations in a modified pBR322 vector. E. coli HB101 was used for transformation. The desired constructs were obtained by a two-step method as described in Materials and Methods. A high level of instability was observed for the plasmid (pRW3348) in which $(\mathrm{CGG})_{160}$ was inserted in orientation II (Figure 4); almost no fragments of the expected size were 


\section{Orientation I Orientation II}

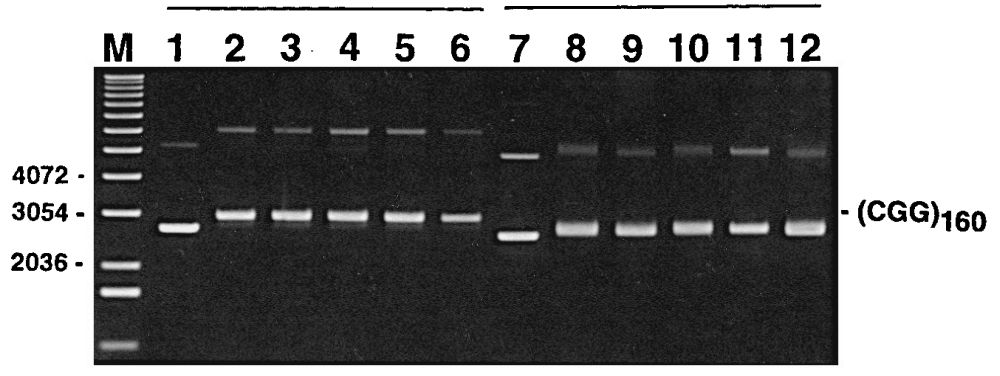

Figure 4. Orientation-dependent stability of a DNA fragment containing $(\mathrm{CGG})_{160}$ in a pBR322 derivative. Five transformants from each orientation were grown in $10 \mathrm{ml}$ and plasmids were extracted and analyzed on a $1 \%$ agarose gel. Lanes 1 and 7 , control plasmids without the CGG insert in orientation I (pRW3342) and in orientation II (pRW3343), respectively; lanes 2 to 6, (CGG) ${ }_{160}$ in pRW3342; and lanes 8 to 12 , in pRW3343. found (lanes 8 to 12). Alternatively, when (CGG) 160 was in orientation I (pRW3347), the insert was stably maintained (lanes 2 to 6). Further analyses of the XbaI/HindIII digests of these plasmids on $5 \%$ PAGE showed the presence of homogeneous (CGG) 160 in pRW3347. Since these plasmids were prepared from a ten hour culture of a single colony on ampicillin plates (no more than 20 generations of E. coli), the orientation of the insert is crucial for maintaining the CGG triplet repeats in a plasmid vector.

As in the case of pRW3348 and 3347, we constructed a plasmid where the (CGG) ${ }_{81}$ insert was cloned in pUC-derived plasmids in both orientations I and II giving pRW3012 and pRW3021, respectively. The recombinant plasmids were then grown in the E. coli SURE strain for $\sim 20$ generations at $37^{\circ} \mathrm{C}$. After isolation of the DNA and double digestion with SacI and HindIII, the products were analyzed on a $1.5 \%$ agarose gel and the bands quantitated. For pRW3012 (orientation I), the insert was $100 \%$ stable whereas in the case of pRW3021 (orientation II), only $43 \%$ of the full-length insert remained and the deletion products ranged from $\sim 10$ to 70 CGG repeats.

The presence of non-CGG triplets (interruptions) in the tandem CGG repeat sequences also influenced the stability. As described above, all plasmids harboring non-interrupted CGGs were cloned into pUC19NotI in orientation II. To test if the instability of pure CGG repeats is also orientation dependent, we recloned the insert from pRW3032 into pUC18-NotI to change the direction of the insert to orientation I. Several deletion products were present in the clone in which a pUC18-Not I derivative contained non-interrupted CGGs in orientation I. Alternatively, 35 repeats containing one CAG interruption and 44 repeats with two interruptions were stably cloned in orientation I, as expected (data not shown).

\section{Two-dimensional gel electrophoresis}

Certain types of simple repeat sequences in plasmids adopt non- $B$ conformations (left-handed Z-DNA, cruciforms, triplexes, etc.) under the influence of negative supercoiling, and the transitions have been investigated by two-dimensional
(2-D) agarose gel electrophoresis (Frank-Kamenetskii \& Mirkin, 1995; Panutin \& Wells, 1992). These structures are stabilized by an underwinding of the primary duplex.

pRW3308, which contains 160 copies of CGG, was analyzed using 2-D gels for both non-methylated and methylated plasmids. Topoisomers were electrophoresed through $1 \%(\mathrm{w} / \mathrm{v})$ agarose in TBE (pH 8.3; Figure 5). Slightly faster-moving spots which are observed in pRW3308 indicate topoisomers in which some CGG repeats were deleted, and are represented as small dots in the lower panel. No insert-specific transition was observed for either unmethylated (left panel) or methylated (right panel) plasmids compared to the vector DNA. Analysis of pRW3310, containing 240 repeats, gave similar results (data not shown).

In addition, plasmids with shorter inserts, 30, 74 and 81 repeats (including interruptions), were investigated by 2-D gel methods under various conditions to explore the potential capacity of the triplet tracts to adopt a non- $B$ structure to obtain higher resolution. Several metal ions in-

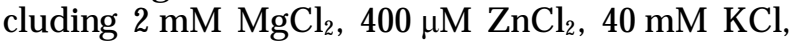
and $150 \mu \mathrm{M} \mathrm{Co}\left(\mathrm{NH}_{3}\right)_{6} \mathrm{Cl}_{3}$ were investigated. Acidic $\mathrm{pH}$ (4.5) was tested also to determine the effect of protonation of cytosine bases in CGGs. None of the gel patterns showed the presence of insertspecific transitions (data not shown).

\section{Discussion}

A series of inserts containing six to 240 copies of CGG were stably cloned in plasmids. Several factors influence the stability (deletions and expansions) of the inserts; repeat length, the presence of interruptions, the orientation of the insert relative to the unidirectional replication origin, E. coli host strains, the location of the insert and the copy number of the host vector. The stability varies strongly with the length of the insert; longer tracts of CGG repeats show a greater degree of instability compared to shorter inserts. This behavior in E. coli is consistent with the mechanism of genetic anticipation for the fragile-X syndrome (Sherman et al., 1985; Fu et al., 1991). Furthermore, the effect of length on DNA polymerase pausing was also observed during synthesis of the repeat in vitro when the Klenow 
fragment of DNA polymerase I was used; lengths of greater than 61 repeats showed stronger pausing sites, occurring at repeat number 30 (away from the CGG start site), when CCG was the template strand. This phenomenon was also observed with CTG triplet repeats (Kang et al., 1995b). These results suggest that, at a critical length, the CGG sequence adopts a non- $B$ conformation(s) which blocks DNA polymerase progression, leading to the idling and subsequent slippage to give expanded products and hence provide the molecular basis for this non-Mendelian genetic process.
The canonical human FMR-1 repeat carries 30 CGG triplets interrupted by two AGG triplets at the tenth and 20th repeat. Fragile- $X$ carriers carry longer repeats (50 to 200) that contain long stretches of uninterrupted CGG triplets which predispose this sequence to hyperexpansion in successive generations. Affected individuals have longer, methylated repeats (230 to 2000; Warren \& Nelson, 1994). Our results indicate that the presence of interruptions greatly enhances the stability of the CGG tract in E. coli. Other studies on alleles derived from human patients show the presence of stable
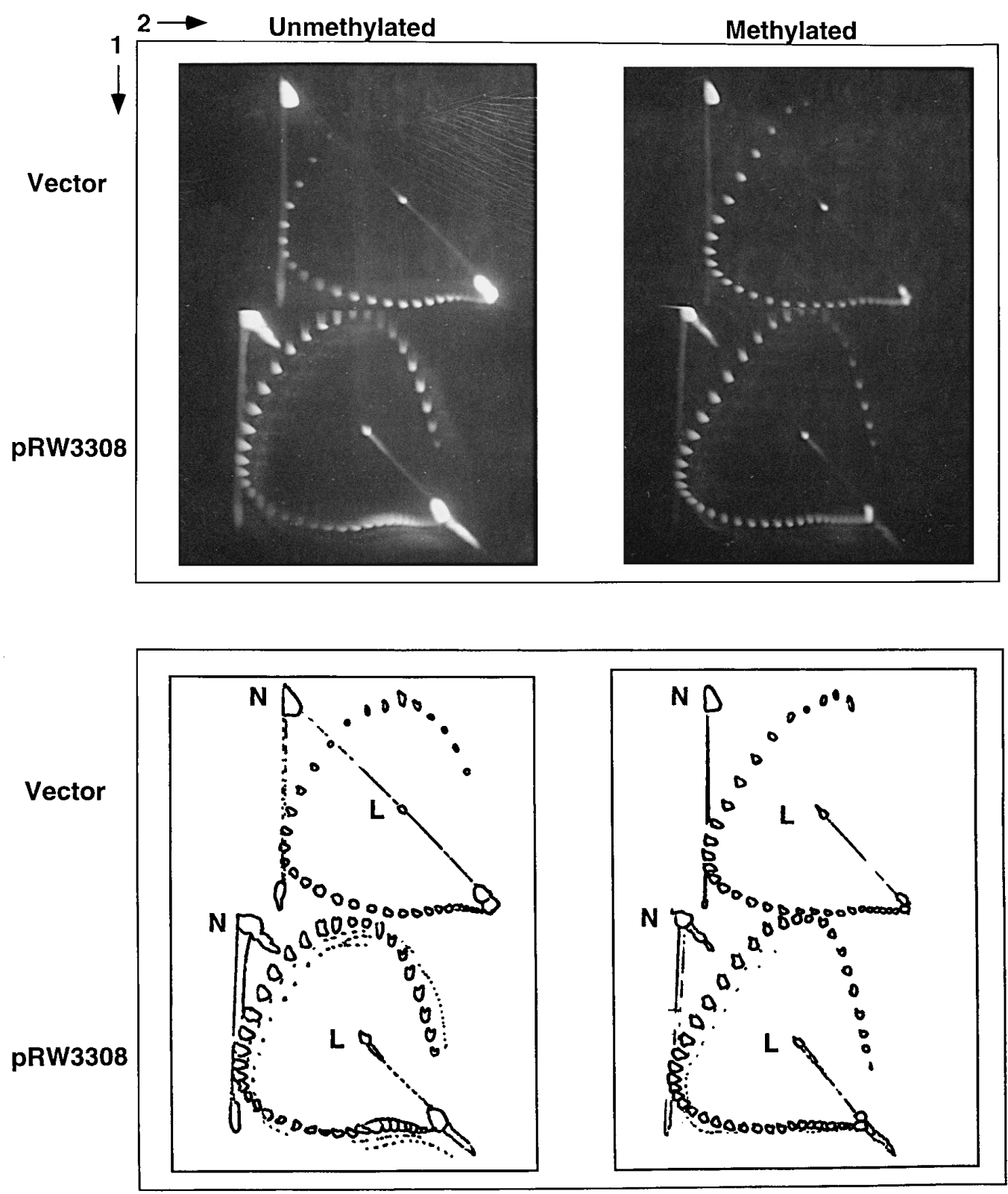

Figure 5. Two-dimensional agarose gel electrophoretic analysis of both unmethylated and methylated pRW3308 and vector (pBR322). Upper panels, gel photographs; lower panels, schematic representations of the gel photographs. Both the vector and the test DNAs were run simultaneously by loading the samples at different positions on the gel. The locations of nicked (N) and linear (L) DNAs are indicated. Open circles represent non-deleted topoisomers, whereas dotted regions for pRW3308 indicate topoisomers in which some CGG repeats have been deleted (see the text). 
and unstable CGG triplets of similar size, suggesting that a feature other than length, but intrinsic to the repeat, was responsible for stability. This supports the observations of Eichler et al. (1994) who found that lengths of $>33$ uninterrupted CGG triplets showed marked instability, regardless of total repeat length, suggesting that the loss of the AGG interruptions is an important mutational event in the generation of alleles predisposed to the fragile- $X$ syndrome.

As mentioned above, another important factor dictating stability is the orientation of the CGG-containing insert. Our results indicate that the triplet repeat was stably maintained in vectors if the CGG strand is in the leading template strand (orientation I) with respect to the origin of replication. However, if CCG falls in the leading template strand (orientation II), the insert was highly destabilized (depending on the length), undergoing deletions and expansions. As in the case of CTG-repeating sequences, the frequency of expansion and deletion of the CGG triplet repeats is influenced by the direction of replication (Kang et al., 1995a) which involves an asymmetric DNA polymerase complex that simultaneously replicates the leading as well as the lagging strands (Wells \& Sinden, 1993). Replication-dependent deletion between direct repeats occurs preferentially in the lagging strand due to the unequal probability of forming hairpins (Trinh \& Sinden, 1991). Therefore, the deletion of the insert (in orientation 1I), can be explained by the propensity of the CGG template strand to form a stable hairpin (Mitas et al., 1995; Chen et al., 1995; Gacy et al., 1995; Mitchell et al., 1995; Fry \& Loeb, 1994) which is bypassed by the replication machinery during resynthesis of the DNA. On the other hand, expansions within the tract are likely due to strand realignment through slippage of the complementary strands during pausing (described above) to generate a folded and elongated nascent DNA on the leading strand (Kang et al., 1995a). Deletions were the most abundant species detected, but expansions were also visible when pRW3024 (i.e. (CGG) $)_{24}$ in orientation II) was propagated in E. coli $\mathrm{DH} 5 \alpha$; the bands differed from each other by one repeating CGG unit suggesting the involvement of slipped structures during replication. This method allowed the cloning of the expanded and deleted products (six to 49 repeats) in orientation I and their propagation in E. coli SURE to give a stable DNA preparation. Studies conducted on the other nine triplet repeats revealed that the CTG sequence has the highest propensity to expand (Ohshima et al., 1996).

Among the strains which were tested (HB101, STB12, and RS2), E. coli SURE was the best choice for stably maintaining the CGG triplet repeats of up to 160 repeats, in pUC-derived plasmids. Inserts, containing more than 160 repeats were extremely unstable in pUC19 and were prone to delete to smaller-sized plasmids. The key for successful cloning for long CGG tracts ( $>160$ repeats) was the two-step method described in the Results. High copy number plasmids offer many advantages as vectors for the cloning and expression of foreign genes but the maintenance of the unstable insert was difficult and deletions were common as described here. Interestingly, in the shuttle vector pGS100 (Sargent et al., 1995) a $12 \mathrm{~kb}$, high copy number, pUC-derived plasmid, $(\mathrm{CGG})_{240}$ was cloned $\sim 1.2 \mathrm{~kb}$ away from the origin of replication and the insert was maintained with a higher stability $(65$ to $75 \%)$ than when cloned into pUC19, where stability dropped to $27 \%$ (data not shown). This result indicates that there are other factors, such as the location of the insert as well as the size of the vector, that influence the stability of long CGG inserts.

Instability of CGG repeats in E. coli is clearly sequence specific, and probably involves two different non- $B$ DNA structures; one is the single-stranded DNA hairpins formed during replication which may be involved in the deletion and expansion events. The other structure is the toroidal conformation present in duplex DNA and is supported by several structural studies including chemical probe analyses (Gellibolian et al., unpublished results). This double-stranded conformation is likely the cause of DNA polymerase pausing within the CGG and CTG repeat tracts (Kang et al., 1995b) and the basis of preferential nucleosome positioning at long CTG repeats (Wang et al., 1994).

Our 2-D gel study revealed no supercoil-induced transitions with the methylated and non-methylated plasmids. Methylation plays an important role in the complete inactivation of the FMR-1 gene in fragile- $X$ patients (Bell et al., 1991; Hornstra et al., 1993). Despite these profound biological differences between the methylated and non-methylated CGG tracts, no structural changes were observed by 2-D gel analyses and by other structural studies such as PAGE (Gellibolian et al., unpublished results). Hence, we conclude that the role of methylation is not at the DNA structural level but must be related to the interactions with the DNA-metabolizing apparatus in vivo.

In summary, the preparation and characterization of a family of plasmids containing (CGG) $n$, where $n=$ six to 240, enables a wide range of investigations on the etiology of fragile- $X$. Despite many studies, few data are available about the mechanism of repeat expansion. The study of repeat (in)stability in vitro or in bacteria is an important tool in understanding the underlying mechanism of repeat instability in humans. Also, cloning in bacteria is the first step towards the introduction of larger repeats in an animal model. In humans, the instability from a normal allele to a disease-causing allele takes many generations (Chakravarti, 1992) and the final step can only take place from a mother to her child. Understanding this mechanism might provide tools for intervention in this step, preventing a premutation from growing to a full mutation which results in disease. 


\section{Materials and Methods}

\section{Enzymes, reagents and strains}

T4 DNA ligase, polynucleotide kinase and Sequenase Version 2.0 were purchased from United States Biochemicals. All restriction endonucleases and Sss I methylase were from New England Biolabs, Inc. Chicken erythrocyte topoisomerase was prepared in this laboratory (gift of J. E. Larson).

Deoxynucleoside 5'-triphosphates were purchased from Boehringer-Mannheim and United States Biochemicals. Chloroquine diphosphate, ethidium bromide and ampicillin sodium salt were from Sigma. Gel electrophoresis was done using I.D.NA agarose from FMC BioProduct for DNA analysis and Long Ranger from J. T. Baker for sequencing. The $1 \mathrm{~kb}$ and $123 \mathrm{bp}$ DNA ladders were purchased from GIBCO BRL. X-gal (5-bromo-4chloro-3-indolyl- $\beta, \mathrm{D}$-galactopyranoside), IPTG (isopropyl$\beta$,D-thiogalactopyranoside), acrylamide and $N$, $N$-methylene-bisacrylamide were purchased from United States Biochemicals: $\left[\gamma^{-32} \mathrm{P}\right]$ ATP was from Amersham. Oligonucleotides were synthesized at the Institute of Biosciences and Technology.

DH5 $\alpha$ (Gibco BRL): $\phi 80$ dlac Z ZM15 $\Delta$ (lacZYA$\arg \mathrm{F}) \mathrm{U} 169$ deo $\mathrm{R}$ rec $\mathrm{A} 1$ end $\mathrm{A} 1$ pho $\mathrm{A} h s d \mathrm{R} 17\left(\mathrm{r}_{\mathrm{K}}^{-}, \mathrm{m}_{\mathrm{K}}^{+}\right) \sup \mathrm{E} 44$ $\lambda^{-}$thi-1 gyrA96 relA1. SURE (Stratagene); $14^{-}\left(\mathrm{McrA}^{-}\right) \Delta$ (morCB-hsdSMR-mrr)171 end A1 sup E44 thi-1 gyrA96 rel $\mathrm{A} 1$ lac rec $\mathrm{B}$ rec $\mathrm{s}$ sc $\mathrm{C}$ umu $\mathrm{C}: \mathrm{T} \mathrm{T} 5\left(\mathrm{Kan}^{\mathrm{r}}\right)$ uvrC $\left(\mathrm{F}^{\prime}\right.$ pro $\mathrm{AB}$ lacI ${ }^{\mathrm{q}} \mathrm{Z} \Delta \mathrm{M} 15$ Tn10 (Tet $\left.{ }^{\mathrm{r}}\right)$ ). HB101 (Gibco BRL): $m c r \mathrm{~B}$ mrr hsd $\mathrm{S} 20\left(\mathrm{r}_{\mathrm{B}}^{-}, \mathrm{m}_{\mathrm{B}}^{-}\right)$rec $\mathrm{A} 13$ sup $\mathrm{E} 44$ ara 14 gal $\mathrm{K} 2$ lac $\mathrm{Y} 1$ pro $\mathrm{A} 2$ rpsL20 $\left(\mathrm{Sm}^{\mathrm{r}}\right) x y l 5 \lambda^{-}$leu mtl 1. STBL2 (Gibco BRL): $m$ cr A $\Delta$ (morBC-hsd RMS-mrr) recA1 end A1 gyrA96 thi sup E44 relA1 $\lambda^{-} \Delta$ (lac-pro $\left.\mathrm{AB}\right)$.

\section{Plasmid construction; construction of plasmids with stretches of pure CGG (method 1)}

pUC19-NotI (Kang et al., 1995a) was used for cloning homogeneous stretches of CGG triplet repeats. A $5 \mu \mathrm{g}$ sample of this plasmid was linearized by digestion with $B a m \mathrm{HI}$ and the DNA was purified as described above. Two duplex oligonucleotides were cloned into this plasmid, giving a total of 24 CGG units. A $2 \mu \mathrm{g}$ (each) sample of oligo 1 (5' GATCCGCTCTTC(GCC) $)_{11} 3^{\prime}$ ) and oligo 2 (3' GCGAGAAG(CGG) ${ }_{11}$ CGGCGG 5') were mixed in one tube in the presence of $25 \mathrm{mM} \mathrm{NaCl}$ and $3 \mathrm{mM}$ $\mathrm{MgCl}_{2}$ in a total volume of $20 \mu \mathrm{l}$. The procedure was repeated for oligo 3 (5' GCCGCC (GCC) ${ }_{11}$ GCGAAGAGC $\left.3^{\prime}\right)$ and oligo 4 (3' (CGG) ${ }_{11}$ CGCTTCTCGCTAG 5'). The samples were treated at $80^{\circ} \mathrm{C}$ and slowly cooled to room temperature over a period of 2.5 hours to allow for efficient annealing. The annealed fragments (FI and FII, Figure 1(a)) were then precipitated with ethanol, washed and dissolved in $15 \mu \mathrm{l}$ of water. FI and FII (150 ng of each) were added to $0.5 \mu \mathrm{g}$ of the linearized vector and ligated with 10 units of T4 DNA ligase for 12 hours at $16^{\circ} \mathrm{C}$ in a volume of $10 \mu \mathrm{l}$. ATP to $1 \mathrm{mM}$ and one unit of polynucleotide kinase were added and the reaction was allowed to proceed at $37^{\circ} \mathrm{C}$ for two hours in an adjusted total volume of $15 \mu \mathrm{l}$.

A $3 \mu \mathrm{l}$ sample of the above ligation mixture was used to electroporate competent E. coli $\mathrm{DH} 5 \alpha$ cells (Dower et al., 1988). A $150 \mu \mathrm{l}$ aliquot of the transformed culture was subsequently plated onto agar plates containing $100 \mu \mathrm{g} / \mathrm{ml}$ of ampicillin, $60 \mu \mathrm{g} / \mathrm{ml}$ of $\mathrm{X}$-gal and $20 \mu \mathrm{g} / \mathrm{ml}$ of IPTG to select, on the basis of color screening, colonies which carry the CGG-containing insert. White colonies were grown in a $10 \mathrm{ml}$ culture in the presence of ampicillin and DNA was isolated by the alkali lysis method (Maniatis et al., 1982). The DNA was characterized by double digestion with SacI and HindIII and the digested products were analyzed on an $8 \%$ polyacrylamide gel in TBE buffer ( $89 \mathrm{mM}$ Tris-borate ( $\mathrm{pH} 8.3$ ), $2 \mathrm{mM}$ EDTA). Clones containing the appropriate-sized insert were selected for sequencing.

\section{Plasmid construction; construction of plasmids with long CGG repeats (method 2)}

pRW3311, pRW3306 and pRW3309 are pUC19-based plasmids and were constructed as follows. The HaeIIIBstUI fragment of RN2, which contains (CGG) (1) , was isolated from a polyacrylamide gel by electroelution and directly subcloned into the HincII site in pUC19. The CGG repeat sequences in RN2, which were derived from the cDNA of fragile-X patients (Verkerk et al., 1991), contain mutations of the perfect repeat at the 12th repeat (AGG) and at the 73rd repeat (CAG) as shown in Table 1. The insert was ligated to generate multimers using $\mathrm{T} 4$ DNA ligase. Longer CGG repeats, (CGG) 160 and (CGG) 240 , are head-to-tail dimers or trimers, respectively, of (CGG) and were also subcloned into pUC19. pRW3306 and pRW3309 also contain non-CGG repeat sequences (CTGGG) at the junction of the two blocks of (CGG) $)_{81}$. pRW3320 and pRW3321 were constructed by recloning the XbaI/HindIII fragment of pRW3311 and pRW3306, respectively, into pUC19NotI (Kang et al., 1995a). For deletion of the CGG repeat sequences, a series of plasmids was constructed based on the reported procedure (Kang et al., 1995a). Briefly, pRW3311, 3306 and 3321 were digested with XbaI and HindIII and run on a polyacrylamide gel. Regions of the gel that were below the major bands (81 or 160 repeating sequences) were electroeluted. The ethanol-precipitated DNA was ligated to XbaI/HindIII-digested pUC19 or pUC19NotI to give pRW3330, pRW3331, pRW3332, pRW3341, pRW3328, pRW3329, pRW3337, pRW3333, pRW3334, pRW3338, pRW3339, pRW3327 and pRW3326 (Table 1). Plasmids were transformed by the calcium chloride method (Maniatis et al., 1982) and grown in SURE cells (Stratagene) for ten hours in the presence of $70 \mu \mathrm{g} / \mathrm{ml}$ ampicillin in LB media with gentle shaking (150 rpm) and, except for pRW3309, were isolated by the cesium chloride method.

pRW3316, pRW3308 and pRW3310 are pBR322-based plasmids and were prepared as follows. The inserts were isolated from pUC19 derivatives (pRW3311, pRW3306 and pRW3309) by cutting the BamHI and HindIII sites of the polylinker. The fragments were cloned into a HindIII and modified EcoRI site in pRW1560 (Jaworski et al., 1989), which is a pBR322 derivative with an 8 bp BglII linker (GAGATCTC) ligated into the filled-in EcoRI site. Plasmids were grown in HB101 in the presence of $70 \mu \mathrm{g} / \mathrm{ml}$ of ampicillin. Since the insert containing 240 copies of CGG was extremely unstable, i.e. prone to deletions, in the pUC19 vector (pRW3309), inserts were isolated from the DNA prepared in small scale $(10 \mathrm{ml}$ culture) by the alkaline lysis method and directly cloned into pBR322 to avoid further deletion of the CGG inserts.

For the study of the orientation-dependent stability of CGG inserts in pBR322, the plasmids which contain $(\mathrm{CGG})_{160}$ in both orientations were constructed. A $322 \mathrm{bp}$ PvuII fragment from pUC19 containing the polylinker was inserted into the filled-in EcoRI and HindIII sites of pBR322 in both orientations to produce pRW3342 (orientation I) and pRW3343 (orientation II); then, the $\mathrm{XbaI}$ and HindIII fragment from pRW3306 containing 


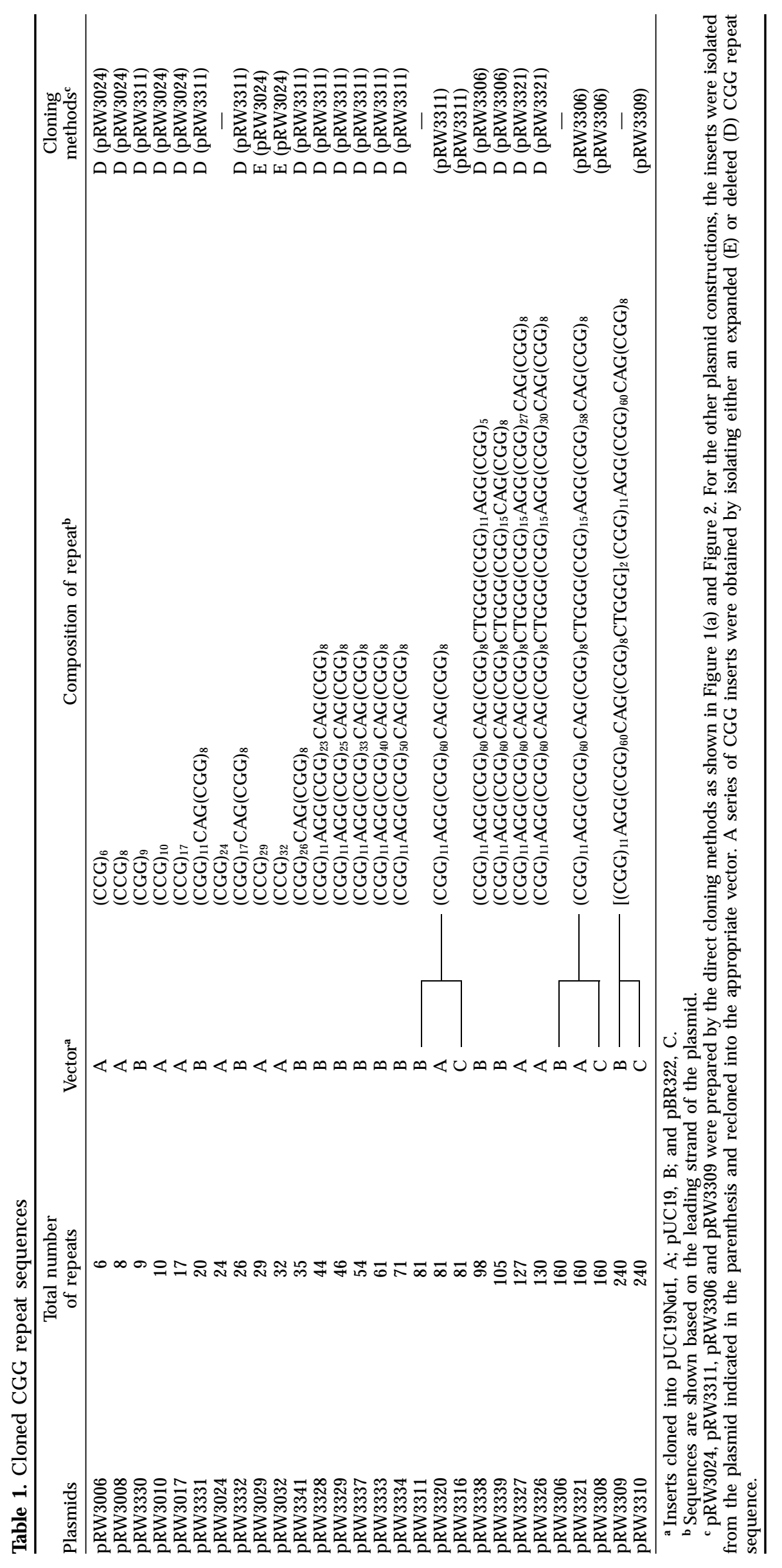


(CGG) $)_{160}$ was ligated into the same restriction site of pRW3342 and pRW3343 to give pRW3347 (orientation I) and pRW3348 (orientation II), respectively.

The integrity of the derivative plasmids and the lengths of their inserts were verified by restriction endonuclease digestion followed by agarose and polyacrylamide gel electrophoresis. Since the CGG containing restriction fragments migrate abnormally fast compared to random sequence DNA (size markers) on non-denaturing polyacrylamide gels (Gellibolian et al., unpublished results), we determined the lengths of the inserts from the results on agarose gels. Complete or partial sequencing was accomplished using the dideoxy chain termination method (see below).

\section{Sequencing of CGG-containing plasmids}

All primers were purchased from New England Biolabs Inc. (M13/pUC sequencing primer (-20), M13/pUC reverse sequencing primer $(-24)$, pBR322 EcoRI site primer (clockwise), and pBR322 HindIII site primer (counter-clockwise)). The $5^{\prime}$ terminus of each primer was radiolabeled using $\left[\gamma-{ }^{32} \mathrm{P}\right] \mathrm{ATP}$ and $\mathrm{T} 4$ polynucleotide kinase. Oligomers were desalted and separated from unincorporated radioactive nucleotides using NENSORB 20 (Dupont) or Sephadex G-50 (Pharmacia). We used the modified method for dideoxy sequencing using Sequenase Version 2.0 to read longer CGG inserts. Denatured plasmid must be freshly made for sequencing; storage of denatured DNA at $-20^{\circ} \mathrm{C}$ caused polymerase pausing during primer extension of CGG-containing plasmids. Plasmids ( 3 to $5 \mu \mathrm{g}$ ) were resuspended in $18 \mu \mathrm{l}$ of water and $2 \mu \mathrm{l}$ of $10 \mathrm{M} \mathrm{NaOH}$ and incubated for 15 minutes at $37^{\circ} \mathrm{C}$. Strong alkaline conditions $(1 \mathrm{M} \mathrm{NaOH})$ were used for complete denaturation. The mixtures were placed on ice and diluted with $132 \mu \mathrm{l}$ of cold water. A $19 \mu \mathrm{l}$ volume of $3 \mathrm{M}$ sodium acetate ( $\mathrm{pH} 5.2)$ was added to neutralize the solution. Denatured plasmids were precipitated with 2.5 volumes of ethanol (at $-80^{\circ} \mathrm{C}$ for several hours). DNA was collected by centrifugation $(16,000 \mathrm{~g}$ for ten minutes at $\left.4^{\circ} \mathrm{C}\right)$ and the pellet was rinsed with $70 \%(\mathrm{v} / \mathrm{v})$ ethanol $(500 \mu \mathrm{l})$ and dried. Use of a primer proximal to the CGG insert is crucial for preventing polymerase pausing during the primer extension reactions. Note that we observed enhanced polymerase pausing inside both the CTG and CGG triplet repeats when a distal primer was used (Kang et al., 1995b). On ice, $5 \mu \mathrm{l}$ ( 2 to $3 \mathrm{ng}$ ) of labeled primer was added to the denatured plasmid and the solution was mixed with $6 \mu \mathrm{l}$ of water and $2 \mu \mathrm{l}$ of $5 \times$ Sequenase reaction buffer $(200 \mathrm{mM}$ Tris- $\mathrm{HCl}(\mathrm{pH}$ 7.5), $100 \mathrm{mM}$ $\mathrm{MgCl}_{2}, 250 \mathrm{mM} \mathrm{NaCl}$ ). The primer was annealed to the denatured DNA by heating the solution at $65^{\circ} \mathrm{C}$ for five minutes, and then slowly cooling to 30 to $37^{\circ} \mathrm{C}$ over a period of 30 minutes (not to exceed one hour). The tube was chilled on ice, and $1 \mu \mathrm{l}$ of $0.1 \mathrm{M}$ DTT and 6.5 units of Sequenase were added. Increasing the deoxynucleotide concentration with respect to the dideoxynucleotide concentration is necessary to extend the sequence further from the primer and to obtain equal intensities of sequencing ladders on all four (A, G, C and T) lanes. The solution $(3.5 \mu \mathrm{l})$ was transferred to $2.5 \mu \mathrm{l}$ of each ddNTP termination mixture $(160 \mathrm{mM}$ dNTPs, and $7.3 \mathrm{mM}$ ddNTP). After ten minutes incubation at $37^{\circ} \mathrm{C}, 4 \mu \mathrm{l}$ of stop solution (95\% (w/v) formamide, $20 \mathrm{mM}$ EDTA, 0.05\% $(\mathrm{w} / \mathrm{v})$ bromophenol blue, and $0.05 \%(\mathrm{w} / \mathrm{v})$ xylene cyanole FF) was added, the DNA was heat denatured at $90^{\circ} \mathrm{C}$ for six minutes, and analyzed by electrophoresis on $10 \%$ (or $8 \%$ ) Long Ranger gels containing 30\% (or $40 \%$ ) formamide and $7 \mathrm{M}$ urea. Autoradiography of the gel was done using either Fuji RX film or Hyperfilm-MP (Amersham). Using this modified method, we could read 100 CCG repeats on the CGG strand and 40 CGG repeats on the CCG strand. The sequences of both complementary strands were determined for all plasmids in Table 1.

\section{Methylation of CGG-containing plasmids}

A $5 \mu \mathrm{g}$ sample of pRW3308 was methylated with 20 units of SssI methylase in the presence of $160 \mathrm{mM}$ $S$-adenosylmethionine in $10 \mathrm{mM}$ Tris- $\mathrm{HCl}$ (pH 7.9), $10 \mathrm{mM} \mathrm{MgCl} 2,50 \mathrm{mM} \mathrm{NaCl}, 1 \mathrm{mM}$ DTT at $37^{\circ} \mathrm{C}$ for 12 hours. If necessary, additional $S$-adenosylmethionine was added to the reaction mixture to obtain a complete reaction. Complete methylation was assessed by digestion with AciI (CCGC/GCGG) which is a methylation-sensitive restriction enzyme which recognizes every CGG repeat sequence. Digestion was analyzed on a $1.0 \%$ agarose gel and the DNA was found to be completely methylated (fully resistant to AciI digestion). Methylated pRW3308 was extracted with phenol and precipitated with ethanol.

\section{Two-dimensional agarose gel electrophoresis}

Topoisomer populations were generated as described previously (Singleton \& Wells, 1982). Both methylated and non-methylated pRW3308 $(6.2 \mu \mathrm{g})$ were incubated in $150 \mu \mathrm{l}$ solution containing $10 \mathrm{mM}$ Tris- $\mathrm{HCl}(\mathrm{pH}$ 7.6), $50 \mathrm{mM} \mathrm{KCl}, 1 \mathrm{mM}$ EDTA, $10 \mathrm{mM}$ 2-mercaptoethanol, and 0 to $12.5 \mu \mathrm{M}$ ethidium bromide. Chicken erythrocyte topoisomerase was added and the reaction was carried out for 12 hours at $37^{\circ} \mathrm{C}$. DNAs were purified by two extractions with phenol and three extractions with ether, and precipitated with ethanol. Topoisomers $(1.5 \mu \mathrm{g} / \mathrm{lane})$ were electrophoresed through $1 \%$ agarose at $3.3 \mathrm{~V} / \mathrm{cm}$ in TBE for 21 hours at room temperature. For the second dimension, gels were run for 18 hours at $3.3 \mathrm{~V} / \mathrm{cm}$ in TBE containing $1 \mu \mathrm{M}$ chloroquine. Gels were stained with ethidium bromide and photographed.

\section{Acknowledgements}

We thank E. Klysik and J. Harris for technical assistance, Dr A. Bacolla for the preparation of several plasmids, and Drs J. Klysik, A. Jaworski, S. Amirhaeri, S. Kang and D. L. Nelson for helpful discussions. This work was supported by the N.I.H. (GM52982) and the Robert A. Welch Foundation.

\section{References}

Ashley, C. T., Jr, Wilkinson, K. D., Reines, D. \& Warren, S. Y. (1993). FMR-1 protein: conserved RNP family domains and selective RNA binding. Science, 262, 563-566.

Bell, M. V., Hirst, M. C., Nakahori, Y., MacKinnon, R. N., Roche, A., Flint, T. J. \& Jacobs, P. A. (1991). Physical mapping across the fragile $\mathrm{X}$ : hypermutation and clinical expression of the fragile $X$ syndrome. Cell, 64, 861-866.

Chakravarti, A. (1992). Fragile X founder effects. Nature Genet. 1, 237-238.

Chen, X., Mariappan, S. V. S., Casasti, P., Ratliff, R., Moyzis, R. K., Laayoun, A., Smith, S. S., Bradbury, E. M. \& Gupta, G. (1995). Hairpins are formed by the 
single DNA strands of the fragile $X$ triplet repeats: structure and biological implications. Proc. Natl Acad. Sci. USA, 92, 5199-5203.

Davies, K. E. \& Warren, S. T. (eds). (1993). Genome Analysis Volume 7: Genome Rearrangement and Stability. Cold Spring Harbor Laboratory Press, Cold Spring Harbor, NY.

Dower, W. J., Miller, J. P. \& Ragsdale, C. W. (1988). High efficiency transformation of $E$. coli by high voltage electroporation. Nucl. Acids Res. 16, 6127-6145.

Eichler, E. E., Holden, J. J. A., Popovich, B. W., Reiss, A. L., Snow, K., Thibodeau, S. N., Richards, C. S., Ward, P. A. \& Nelson, D. L. (1994). Length of uninterrupted CCG repeats determines instability in the FMR-1 gene. Nature Genet. 8, 88-94.

Eichler, E. E., Hammond, H. A., Macpherson, J. N., Ward, P. A. \& Nelson, D. L. (1995). Population survey of the human FMR1 CGG repeat substructure suggests biased polarity of the loss of AGG interruptions. Hum. Mol. Genet. 4, 2199-2208.

Frank-Kamenetskii, M. D. \& Mirkin, S. M. (1995). Triplex DNA structures. Annu. Rev. Biochem. 64, 65-95.

Fry, M. \& Loeb, L. A. (1994). The fragile X syndrome $\mathrm{d}(\mathrm{CGG})_{\mathrm{n}}$ nucleotide repeats form a stable tetrahelical structure. Proc. Natl Acad. Sci. USA, 91, 4950-4954.

Fu, Y.-H., Kuhl, D. P. A., Pizzuti, A., Pieretti, M., Sutcliffe, J. S., Richards, S., Verkerk, A. J. M. H., Holden, J. J. A., Fenwick, R. G., Jr, Warren, S. T., Oostra, B. A., Nelson, D. L. \& Caskey, C. T. (1991). Variation of the CGG repeat at the fragile $X$ site results in genetic instability: resolution of the Sherman paradox. Cell, 67, 1047-1058.

Gacy, A. M., Goellner, G., Juranic, N., Macura, S. \& McMurray, C. T. (1995). Trinucleotide repeats that expand in human disease form hairpin structures in vitro. Cell, 81, 533-540.

Gastier, J. M., Pulido, J. C., Sunden, S., Brody, T., Buetow, K. H., Murray, J. C., Weber, J. L., Hudson, T. J., Sheffield, V. C. \& Duyk, G. M. (1995). Survey of trinucleotide repeats in the human genome: assessment of their utility as genetic markers. Hum. Mol. Genet. 4, 1829-1836.

Hornstra, I. K., Nelson, D. L., Warren, S. T. \& Yang, T. P. (1993). High resolution methylation analysis of the FMR-1 gene trinucleotide repeat region in fragile $X$ syndrome. Hum. Mol. Genet. 2, 1659-1665.

Jaworski, A., Blaho, J. A., Larson, J. E., Shimizu, M. \& Wells, R. D. (1989). Tetracycline promoter mutations decrease non B DNA structural transitions, linking numbers and deletions in recombinant plasmids in Escherichia coli. J. Mol. Biol. 207, 513-526.

Jones, C., Penny, L., Mattina, T., Yu, S., Baker, E., Voullaire, L., Langdon, W. Y., Sutherland, G. R., Richards, R. I. \& Tunnacliffe, A. (1995). Association of a chromosome deletion syndrome with a fragile site within the proto-oncogene CBL2. Nature, 376, 145-149.

Kang, S., Jaworski, A., Ohshima, K. \& Wells, R. D. (1995a). Expansion and deletion of CTG triplet repeats from human disease genes are determined by the direction of replication. Nature Genet. 10, 213-218.

Kang, S., Ohshima, K., Shimizu, M., Amirhaeri, S. \& Wells, R. D. (1995b). Pausing of DNA synthesis in vitro at specific loci in CTG and CGG triplet repeats from human hereditary diseases. J. Biol. Chem. 270, 27014-27021.

Klysik, J., Stirdivant, S. M. \& Wells, R. D. (1982). Left handed DNA cloning, characterization and instabil- ity of inserts containing different lengths of (dC-dG) in Escherichia coli. J. Biol. Chem. 257, 10152-10158.

Knight, S. J. L., Flannery, A. V., Hirst, M. C., Campbell, L., Christodoulou, Z., Phelps, S. R., Pointon, J., Middleton-Price, H. R., Oostra, B. A. \& Davies, K. E. (1993). Trinucleotide repeat amplification and hypermethylation of a CPG island in FRAXE mental retardation. Cell, 74, 127-134.

Kremer, E. J., Pritchard, M., Lynch, M., Yu, S., Holman, K., Baker, E., Warren, S. T., Schlessinger, D., Sutherland, G. R. \& Richards, R. I. (1991). Mapping of DNA instability at the fragile $X$ to a trinucleotide repeat sequence $\mathrm{p}(\mathrm{CCG})_{\mathrm{n}}$. Science, 252, 1711-1714.

Lukomski, S. \& Wells, R. D. (1995). Left-handed ZDNA and in vivo supercoil density in the E. coli chromosome. Proc. Natl Acad. Sci. USA, 91, 99809984.

Maniatis, T., Fritsch, E. F. \& Sambrook, J. (1982). Molecular Cloning: A Laboratory Manual. Cold Spring Harbor Laboratory Press, Cold Spring Harbor, NY.

Mitas, M., Yu, A., Dill, J. \& Haworth, I. S. (1995). The trinucleotide repeat sequence $\mathrm{d}(\mathrm{CGG})_{15}$ forms a heat-stable hairpin containing $G^{\text {syn }} \cdot G^{\text {anti }}$ base pairs. Biochemistry, 34, 12803-12811.

Mitchell, J. E., Newbury, S. F. \& McClellan, J. A. (1995). Compact structures of $\mathrm{d}(\mathrm{CNG})_{\mathrm{n}}$ oligonucleotides in solution and their possible relevance to fragile $\mathrm{X}$ and related human genetic diseases. Nucl. Acids Res. 23, 1876-1881.

Nelson, D. L. (1993). Six human genetic disorders involving mutant trinucleotide repeats. In Genome Analysis Volume 7: Genome Rearrangement and Stability (Davies, K. E. \& Warren, S. T., eds), pp. 1-24, Cold Spring Harbor Laboratory Press, Cold Spring Harbor, NY.

Ohshima, K., Kang, S. \& Wells, R. D. (1996). CTG triplet repeats from human hereditary disease are dominant genetic expansion products in E. coli. J. Biol. Chem. 271, 1853-1856.

Panyutin, I. \& Wells, R. D. (1992). Nodule DNA in the $(\mathrm{GA})_{37} \cdot(\mathrm{CT})_{37}$ insert in superhelical plasmids. J. Biol. Chem. 267, 5495-5501.

Reyniers, E., Vits, L., De Boulle, K., Van Roy, B., Van Velzen, D., de Graaff, E., Verkerk, A. J. M. H., Jorens, J. Z. J., Darby, J. K., Oostra, B. A. \& Willems, P. J. (1993). The full mutation in the FMR-1 gene of male fragile $X$ patients is absent in their sperm. Nature Genet. 4, 143-146.

Sargent, R. G., Merrihew, R. V., Nairn, R., Adair, G., Meuth, M. \& Wilson, J. H. (1996). The influence of a $(\mathrm{GT})_{29}$ microsatellite sequence on homologous recombination in the hamster APRT gene. Nucl. Acids Res. 24, 746-753.

Sherman, S. L., Jacobs, P. A., Morton, N. E., FrosterIskenius, U., Howard-Peebles, P. N., Nielsen, K. B., Partington, M. W., Sutherland, G. R., Turner, G. \& Watson, M. (1985). Further segregation analysis of the fragile $X$ syndrome with special reference to transmitting males. Hum. Genet. 69, 289-299.

Sinden, R. R. (1994). DNA Structure and Function. Academic Press, San Diego, CA.

Sinden, R. R. \& Wells, R. D. (1992). DNA structure, mutations, and human genetic disease. Curr. Opin. Biotech. 3, 612-622.

Singleton, C. K. \& Wells, R. D. (1982). The facile generation of covalently closed circular DNAs with defined negative superhelical densities. Anal. Biochem. 122, 253-257.

Siomi, H., Siomi, M. C., Nussbaum, R. L. \& Dreyfuss, G. (1993). The protein product of the fragile $X$ gene, 
FMR1, has characteristics of an RNA-binding protein. Cell, 74, 291-298.

Sutcliffe, J. S., Nelson, D. L., Zhang, F., Pieretti, M., Caskey, C. T., Sachs, D. \& Warren, S. T. (1992). DNA methylation represses FMR-1 transcription in fragile X syndrome. Hum. Mol. Genet. 1, 397-400.

Trinh, T. Q. \& Sinden, R. R. (1991). Preferential DNA secondary structure mutagenesis in the lagging strand of replication in E. coli. Nature, 352, 544-547.

Verkerk, A. J. M. H., Pieretti, M., Sutcliffe, J. S., Fu, Y.-H., Kuhl, D. P. A., Pizzuti, A., Reiner, O., Richards, S., Victoria, M. F., Zhang, F., Eussen, B. E., van Omen, G. J. B., Blonden, L. A. J., Riggins, G. J., Chastain, J. L., Kunst. C. B., Galjaard, H., Caskey, C. T., Nelson, D. L., Oostra, B. A. \& Warren, S. T. (1991). Identification of a gene (FMR-1) containing a CGG repeat coincident with a breakpoint cluster region exhibiting length variation in fragile $X$ syndrome. Cell, 65, 905-914.

Wang, Y.-H., Amirhaeri, S., Kang, S., Wells, R. D. \& Griffith, J. (1994). DNA triplet repeats from the myotonic dystrophy gene are preferential nucleosome assembly sites in vitro. Science, 265, 669-671.
Warren, S. T. \& Nelson, D. L. (1994). Advances in molecular analysis of fragile $\mathrm{X}$ syndrome. J. Am. Med. Assoc. 271, 536-542.

Wells, R. D. (1996). Molecular basis of genetic instability of triplet repeats. J. Biol. Chem. 271, 2875-2878.

Wells, R. D. \& Sinden, R. R. (1993). Defined ordered sequence DNA, DNA structure and DNA-directed mutation. In Genome Analysis, Volume 7: Genome Rearrangement and Stability (Davies, K. E. \& Warren, S. T., eds), pp. 107-138, Cold Spring Harbor Laboratory Press, Cold Spring Harbor, NY.

Wohrle, D., Hennig, I., Vogel, W. \& Steinbach, P. (1993). Mitotic stability of fragile $X$ mutations in differentiated cells indicates early post-conceptional trinucleotide repeat expansion. Nature Genet. 4, 140-142.

Yu, S., Mulley, J., Loesch, D., Turner, G., Donnelly, A., Gedeon, A., Hillen, D., Kremer, E., Lynch, M., Pritchard, M., Sutherland, G. R. \& Richards, R. I. (1992). Fragile $X$ syndrome: unique genetics of the heritable unstable element. Hum. Genet. 50, 968-980

Edited by J. Karn

(Received 27 October 1995; received in revised form 9 February 1996; accepted 13 February 1996) 